Sheridan College

SOURCE: Sheridan Institutional Repository

$5-2009$

\title{
Canine Pose Estimation: A Computing for Public Safety Solution
}

\author{
Cristina Ribeiro \\ Sheridan College, cristina.ribeiro@sheridancollege.ca \\ Alexander Ferworn \\ Ryerson University \\ Mieso Denko \\ University of Guelph \\ James Tran \\ Ryerson University
}

Follow this and additional works at: https://source.sheridancollege.ca/fast_publications

Part of the Computer Engineering Commons

\section{SOURCE Citation}

Ribeiro, Cristina; Ferworn, Alexander; Denko, Mieso; and Tran, James, "Canine Pose Estimation: A Computing for Public Safety Solution" (2009). Publications and Scholarship. 50.

https://source.sheridancollege.ca/fast_publications/50

\section{(c) (1) (9)}

This work is licensed under a Creative Commons Attribution-Noncommercial-No Derivative Works 4.0 License. This Conference Paper is brought to you for free and open access by the Faculty of Applied Science \& Technology (FAST) at SOURCE: Sheridan Institutional Repository. It has been accepted for inclusion in Publications and Scholarship by an authorized administrator of SOURCE: Sheridan Institutional Repository. For more information, please contact source@sheridancollege.ca. 


\section{Canine Pose Estimation: A Computing for Public Safety Solution}

\author{
Cristina Ribeiro \\ Department of \\ Computing and \\ Information Science, \\ University of Guelph, \\ 50 Stone Road, \\ Guelph, Ontario, \\ N1G 2W1, Canada \\ cribeiro@uoguelph.ca
}

\author{
Alexander Ferworn \\ Department of Computer \\ Science, \\ Ryerson University, \\ 350 Victoria Street, \\ Toronto, Ontario, \\ M5B 2K3, Canada \\ aferworn@scs.ryerson.ca
}

\author{
Mieso Denko \\ Department of \\ Computing and \\ Information Science, \\ University of Guelph, \\ 50 Stone Road, \\ Guelph, Ontario, \\ N1G 2W1, Canada \\ mdenko@uoguelph.ca
}

\author{
James Tran \\ Department of \\ Computer Science, \\ Ryerson University, \\ 350 Victoria Street, \\ Toronto, Ontario, M5B \\ 2K3, Canada \\ q2tran@scs.ryerson.ca
}

\begin{abstract}
In this paper we discuss determining canine pose in the context of common poses observed in Urban Search and Rescue dogs through the use a sensor network made up of accelerometers. We discuss the use of the Canine Pose Estimation System in a disaster environment, and propose techniques for determining canine pose. In addition we discuss the challenges with this approach in such environments. This paper presents the experimental results obtained from the Heavy Urban Search and Rescue disaster simulation, where experiments were conducted using multiple canines, which show that angles can be derived from acceleration readings. Our experiments show that similar angles were measured for each of the poses, even when measured on multiple USAR canines of varying size. We also developed an algorithm to determine poses and display the current canine pose to the screen of a laptop. The algorithm was successful in determining some poses and had difficulty with others. These results are presented and discussed in this paper.
\end{abstract}

\section{Introduction}

Urban Search and Rescue (USAR) is a difficult, time consuming and strenuous undertaking for humans [1]. Often canines are employed in the search because of their agility, speed and strong sense of smell. While their agility is an asset to USAR it is also a potential impediment for canine handlers as the handler is not as fast or as agile as the dog [1,2]. As a result, the handlers and other emergency responders are sometimes unaware of the canine's actions and orientation [3,4]. The Network-Centric Applied Research Team (N-CART) at Ryerson University is developing Canine Augmentation Technology (CAT), adding technology components to canines in order to improve the interaction between the dog and rescuers. The CAT system is equipped with wireless pan and tilt cameras mounted on each shoulder of the canine. This enables rescuers to view the disaster site from the canine's perspective without entering the unsafe zone.

We have achieved some success culminating in our participation in a large structural collapse exercise held by Canada Task Force 3 (Toronto) in June 2007. CAT took valuable footage of the surrounding disaster area within a space that human were not allowed to enter, including a picture of a casualty. One area, which is lacking is the ability to know what the dog is actually doing when the video is taken. This information is important because it is often difficult to have sufficient situational awareness of what is going on with the dog when one does not have a grasp of what the dog is going through and in what way it has aligned its body to achieve a particular shot.

The Canine Pose Estimation (CPE) System determines pose through the use of technology and provides interested individuals with the dog's current body position. This has implications for a variety of search situations when the canine is working in extremely confined spaces and it cannot be directly observed. From pose information it may be possible to determine clues about the situation of any discovered human casualties. The canines are trained to indicate different events employing both sound (barking) and body position (pose).

For example, the canine may be in the sitting position - an indication that the dog has found a cadaver. When the canine is standing or lying down, this indicates when the dog actively searching (in the standing pose) or not (lying down pose). This paper looks at reproducible patterns in data collected for each of the poses. The closest work that was done in our research area was conducted in [5]. However, this 
research was conducted for one feline and using only one accelerometer. They studied patterns arising from the acceleration readings using a fast Fourier transform algorithm. In our work we use acceleration to determine angles, and use the angles to devise an algorithm to determine canine pose. Our experiments include two dual axis accelerometers mounted on multiple canines.

The rest of this paper is organized as follows: Section 2 presents an overview of USAR, its implementation, some challenges and the use of accelerometers to determine canine pose. Section 3 discusses different ways to determine canine pose. Section 4 presents, a description of the methods and materials used and communication challenges. Section 5 presents discussion of the experimental results. Finally, section 6 presents the conclusion and discusses future research directions.

\section{Related Work}

Service dogs exist all around us, many already carrying a variety of technology on them. One example is the PetsCell, a cell phone for dogs with GPS, which enables the owner to track their dog [6]. Another example is FIDO, adopted by various UK police forces, a camera system for police canines involved in arrests, specifically for weapons seizures [7]. When it comes to search and rescue, it would be helpful to know more about what the dog is experiencing in terms of orientation and position to achieve better situational awareness (SA). We call this pose determination.

Situational awareness has been shown to be a problem in a number of fields including Human Robot Interaction (HRI) with USAR response robots. The problem is that the robot operators often do not have a direct view of the robot and rely solely on the robot's cameras for SA. For the most part, the operators look outward and do not have access to self-views. In [8, 9] it was shown that operators spent on average $30 \%$ of their time on SA activity. It was found that they had less SA of the space behind the robot in comparison to the space in front or on the sides of the robot. They have encountered difficulty in maintaining SA when in autonomous mode.

Often rescuers cannot determine where the up position is, making it extremely difficult to discern the camera's orientation. This is an especially difficult problem with regard to the use of canines, as their agility allows them to twist into very small cavities in rather odd orientations. On occasion it may be important to know what the dog is doing in order to give it further instructions when it can still hear its handler but cannot see him. For example, a USAR dog may become interested by a certain scent that does not relate to finding a casualty. It would be useful for the handler to know that the dog is stopped and has his head down. This information about the dog is difficult to obtain since no one can see the dog and placing a camera on the dog in order to see the dog is not feasible as there is no obvious way of doing this.

\section{Canine pose estimation}

Search canines posses superior agility and speed compared to its handlers, rubble searches are conducted off-leash. USAR canines are adept at moving around alone and are focused on achieving the task of finding people buried in rubble and usually requires little guidance; the dogs act as an autonomous agent on the rubble pile while searching. As the dogs make decisions on the pile, they may move beyond the range within which the handlers can control the dogs through visual and spoken commands and signals.

This creates a problem since the handler may not hear the canines or see its pose in the event that the dogs find a patient. Since the dogs are also trained to stay where the patient is located an obvious problem exists with interacting with the dogs. It is during these circumstances that knowing the canine's pose would be a significant asset to the handler and those interested in the progress of the search. To solve this problem a CPE device was designed to provide a canine's pose status to the handler.

The CPE algorithm that was developed based on the training data collected in the first set of experiments. This telemetric algorithm automatically measures two angles from two reference points on the dog. The CPE software on the laptop then transmits the data wirelessly for recording and analysis. The CPE algorithm estimates four poses including: standing, lying down, sitting, and walking. These poses are discussed in detail in the following sections.

\subsection{Defining the standing pose}

A schematic diagram of a canine standing showing the measured acceleration for this pose can be found in Figure 3.1. In this diagram the acceleration gravity vector is parallel to the $\mathrm{Y}$ axis of the accelerometer, which translates into a zero degree angle for both accelerometers on the dog.

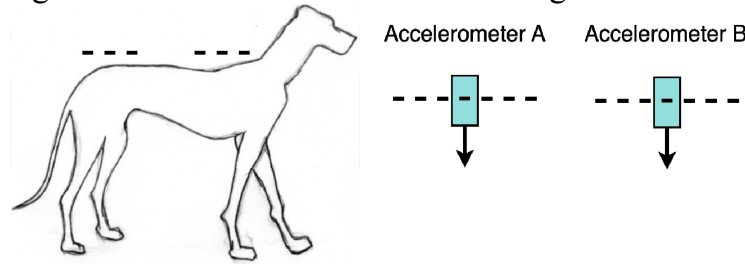

Figure 3.1. Standing pose schematic diagram 
Figure 3.2 and 3.3 is the graphical representation of the data collected from USAR canines in the standing pose. This pose was determined when angle A and angle B were simultaneously $+/-5$ degrees from the previous angle measurement. This time window for this case was set at 500 milliseconds before determining this pose. For this pose it was observed that some dogs were very still. In other tests, the dogs wagged their tail and moved their head, looking around and up at their handler. Even in this case the observed amplitude of the angles did not exceed $+/-5$ degrees.

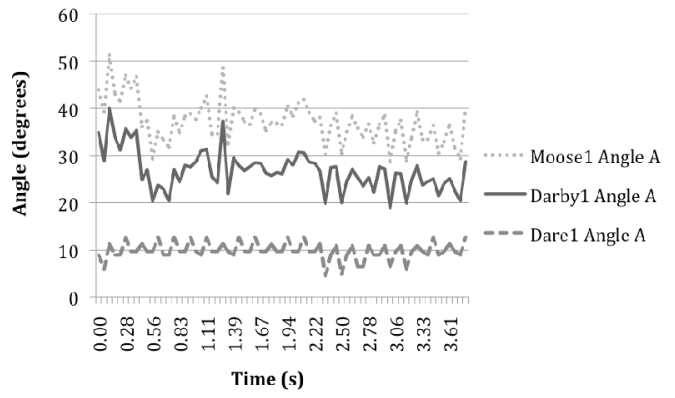

Figure 3.2. Data collected from USAR canines standing angle $A$

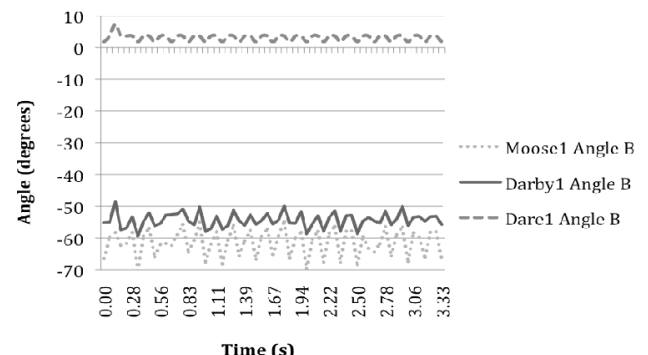

Figure 3.3. Data collected from USAR canines standing angle $B$

\subsection{Defining the sitting pose}

In Figure 3.4 is a schematic diagram of a canine sitting. This diagram shows an arrow representing the acceleration gravity vector reading on the accelerometer. Angle A and B are slightly different in their readings, this is shown in Figure 3.4 by the different slopes of the dashed lines.

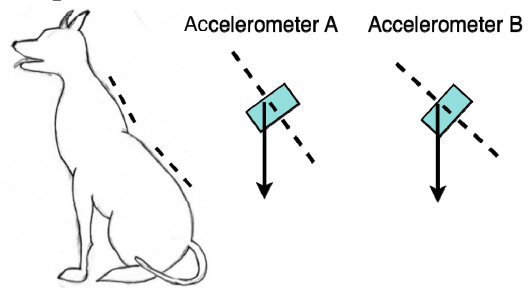

Figure 3.4. Sitting pose schematic diagram

The sitting pose required two sets of rules to distinguish between two different sitting styles that were observed among the USAR dogs used in the study. In the first case, a crossing over was identified where angle B became increasingly larger than angle A, shown in Figure 3.5. Moreover, the difference between angle $\mathrm{A}$ and $\mathrm{B}$ was between 10 and 40 degrees. This range can be seen clearly in Figure 3.5. The crossing over of the angles was observed when the canine started to sit on its hind legs and as such angle $\mathrm{B}$ became larger than angle $\mathrm{A}$.

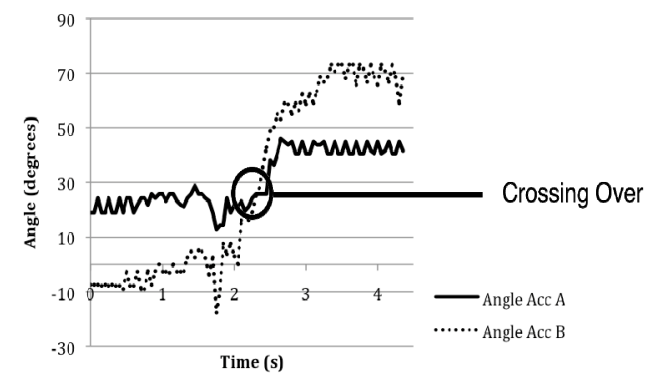

\section{Figure 3.5. Data collected from USAR canine Moose sitting}

The second sitting case was observed when angle $\mathrm{A}$ and $\mathrm{B}$ increased or decreased simultaneously as shown in Figure 3.6. This was classified as a crossing over and crossing back condition in the CPE algorithm. In this case as the dog sat on its hind legs, angle B increased in amplitude greater than angle A; however, this amplitude was not as great as in the first sitting style.

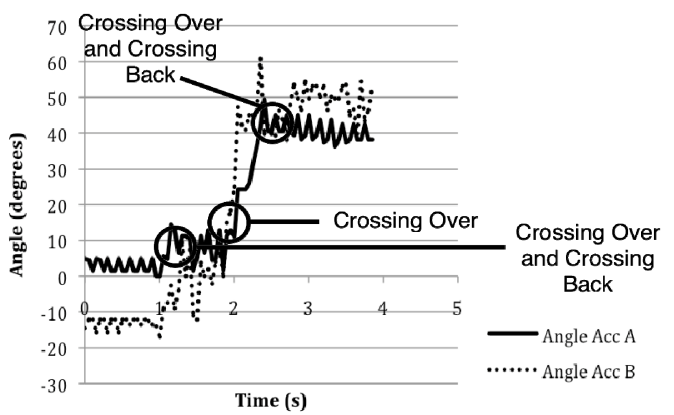

Figure 3.6. Data collected from USAR canine Darby sitting

It was observed that the crossing back occurred because of the shorter length of the dog's back, which resulted in the accelerometers being strapped closer to each other on the dog. In addition, after the crossing back occurred, it was recognized that the difference between the angles was found to be less than 15 degrees and the cumulative angle was greater than 30 degrees. This was observed to be the range of the angles measured for the canines tested, as shown in Figures 3.7 and 3.8. 


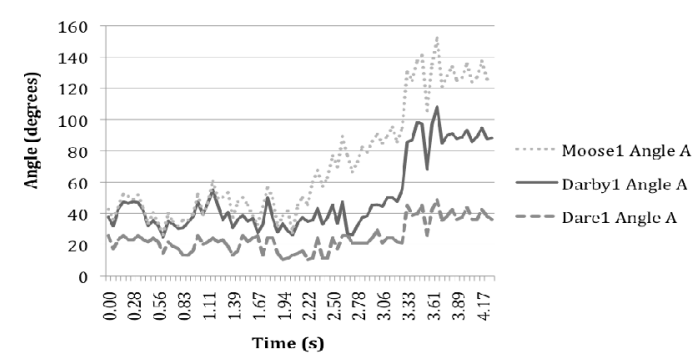

Figure 3.7. Data collected from USAR canines sitting angle $A$

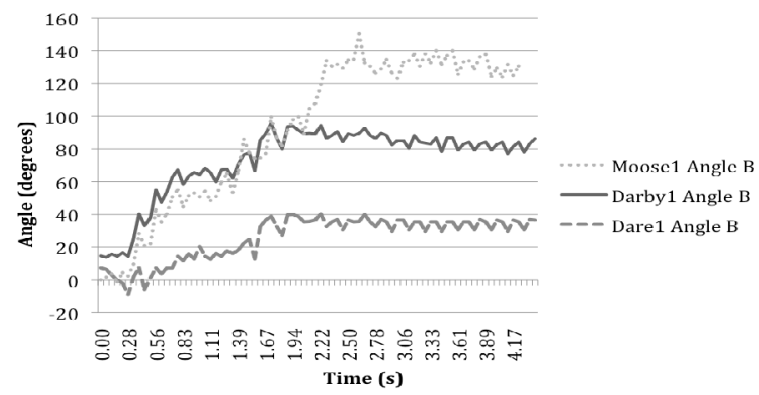

Figure 3.8. Data collected from USAR canines sitting angle $B$

\subsection{Defining the lying down pose}

A schematic diagram of a canine lying down is shown in Figure 3.9. The accelerometers are shown to have slightly different angle readings. This can be seen in more detail in Figure 3.9.

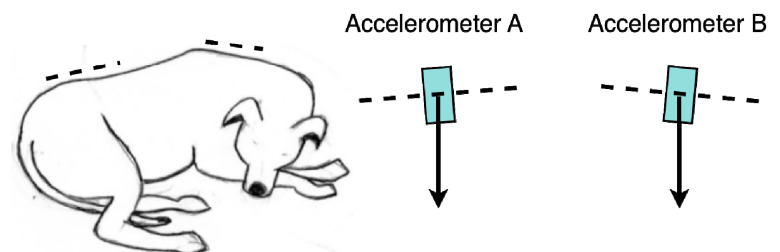

Figure 3.9. Lying down pose schematic diagram

The lying down pose was distinguished by an increase in angle $\mathrm{A}$ and angle $\mathrm{B}$ with combined amplitude of greater than 35 degrees but less than 95 degrees as shown in Figure 3.10. In addition, a second condition was observed where angle $\mathrm{B}$ was greater than or equal to -10 degrees and less than 95 degrees.

Analysis of the angles when the canine starts to lie down showed that both angles increase simultaneously. When the dog performed this pose it started to sit on its hind legs, which caused an increase in both angles; similar to what was observed for the sitting pose. The range in amplitude of the angles was discovered to be between 35 and 95 degrees, when comparing the data from all of the tested dogs.

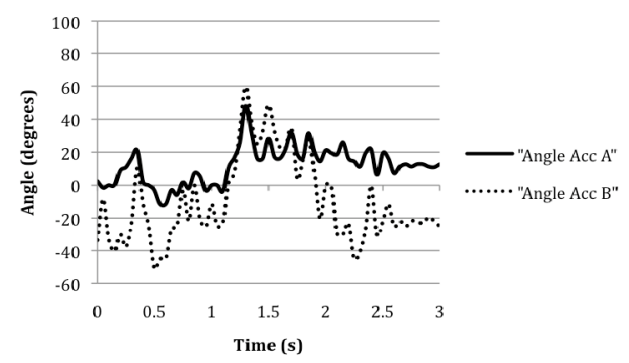

Figure 3.10. Data collected from USAR canine Dare lying down

The second condition that was observed occurred when the dog slid its front legs down to complete the pose. It was found that both angles decreased with this movement and that angle B was consistently in the range of -10 and 95 degrees when the canine had completed the pose. Each of these conditions occurred for a minimum of 500 milliseconds. These conditions were observed as demonstrated in the data collected from the primary series of experiments in Figures 3.11 and 3.12 .

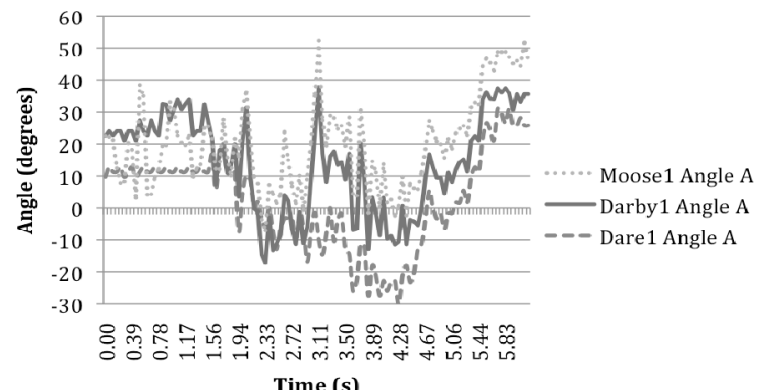

Figure 3.11. Data Collected from USAR Canines Lying Down Angle A

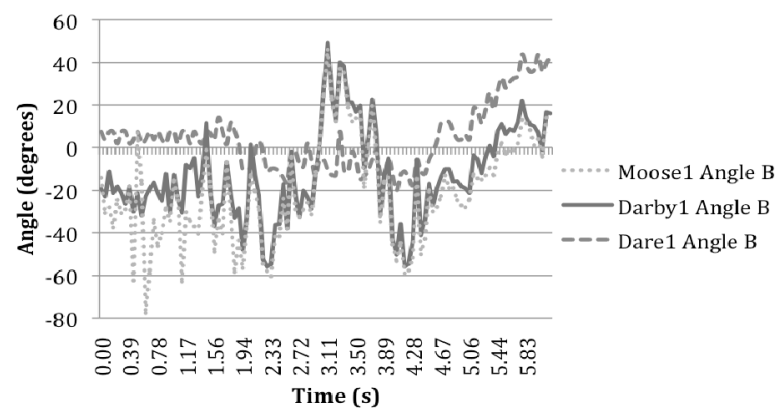

Figure 3.12. Data collected from USAR canines lying down angle $B$

\subsection{Defining the walking pose}

This pose is represented in a schematic diagram shown in Figure 3.13. The way the accelerometers were strapped onto the canine the angle readings originally were horizontal as found in the standing 
pose Figure 3.13. When the dog walked its body was in motion and the angle readings varied; this can be seen in more detail in Figure 3.14.

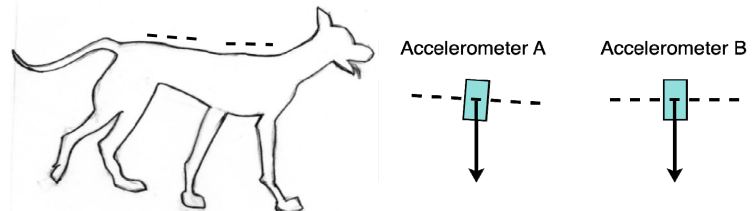

Figure 3.13. Walking pose schematic diagram

The walking pose was distinguished by both angles' amplitudes being greater than 15 degrees when compared to the previous sample. This is shown in Figure 3.14. This pattern was similar to the standing pose except that the amplitude of both angles was much greater for this pose. The pattern occurred consecutively for a minimum of 500 milliseconds. The angle range was uniform for all experiments conducted for the walking pose regardless of the canine tested, as shown in Figures 3.15 and 3.16.

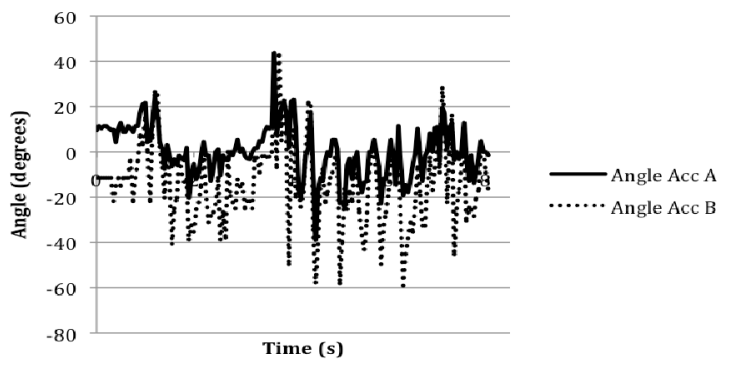

Figure 3.14. Data collected from USAR canine Moose walking

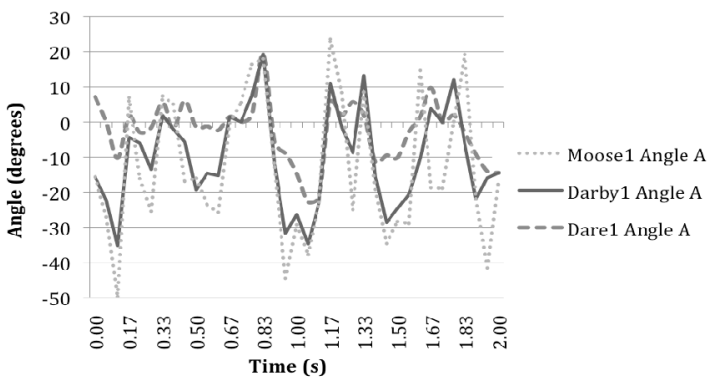

Figure 3.15. Data collected from USAR canines walking angle $A$

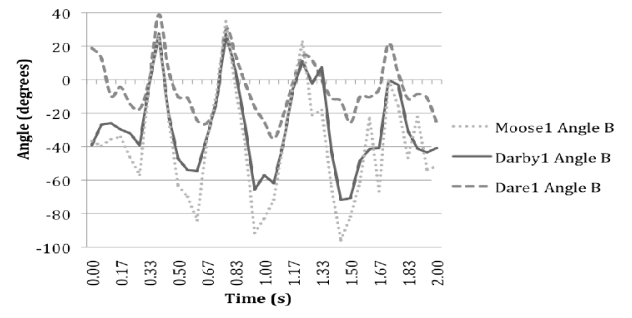

Figure 3.16. Data collected from USAR canines walking angle $B$

\section{Method}

A series of experiments were used to assess the predictive accuracy of the CPE algorithm. The analysis of the results obtained led to the performance evaluation of the CPE algorithm. These experiments were conducted on a total of five USAR dogs. Each test involved applying the algorithm to each of the four different poses by each of the dogs.

Experiments were conducted at the Ontario Provincial Police USAR structural collapse training site in Bolton with the Provincial Emergency Response Teams - Canine Unit in September 2008. Both these series of experiments and the preliminary experiments where we collected training data used to develop the CPE algorithm, were conducted using the same procedure as outlined below.

Prior to a test beginning, each USAR canine was fitted with the harness containing the CPE device. The CPE device was not calibrated for each canine. The sensors in the CPE device secured accelerometer A on the dorsal vertebrae (wither), which is near the head of the canine, and accelerometer B was affixes on the lumbar vertebrae (loin), which is near the tail of the dog. The harness and the CPE device is affixed on the dog as shown in Figure 4.1.

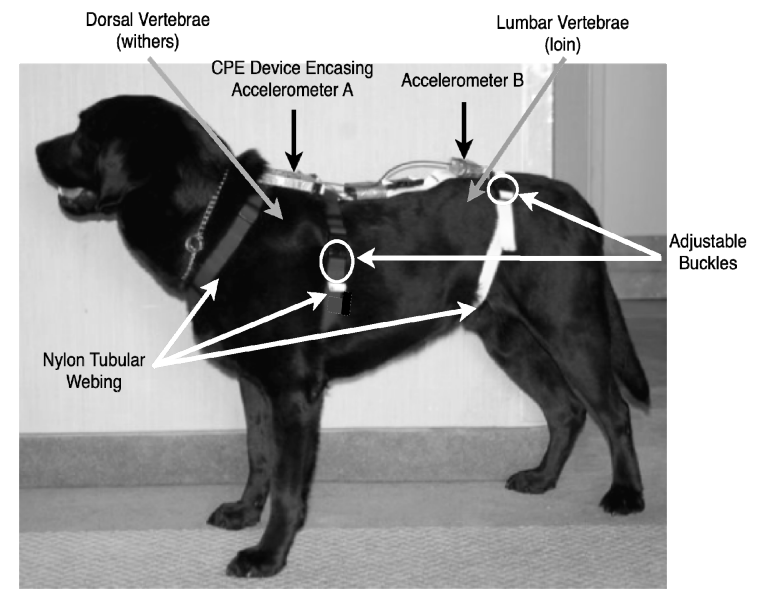

Figure 4.1. USAR canine Dare wearing the CPE device

The pose being recorded was told to the canine handler in advance so that they were prepared to command their dog. In order to synchronize the data being collected with the filming of the test, the videographer readied the camera and signaled the test to begin and the person with the laptop started the application simultaneously. The handler then gave the pose commands to the canine and the results were recorded by the application. 
The application captured the following: 1. the raw accelerometer data, 2 . all the calculations made to determine pose, 3 . the pose estimated and 4. a time stamp for each data set received from the CPE device. This data was written to a file and saved for data analysis. The pre-processing of the raw accelerometer data involved confirming that the data was not corrupted. The data was checked for the expected four digit analog accelerometer readings. If the data was garbled with random bits the data was considered corrupt and unusable and the test would have been thrown out. The data was confirmed as a complete data string if the string bit identifier *, was recorded before any accelerometer data was recorded.

Access to USAR canines and their handlers was infrequent as they were engaged in many different search scenarios during the exercises. When conducting experiments the USAR teams were available for only a few hours. It took 30 minutes of set up time per USAR team for each experiment. The average time taken to complete each experiment with five tests per pose was one hour per dog involved in the experiment. These time restrictions reduced the number of tests that were physically possible. The total number of dogs experimented with was 5 .

\section{Experimental results and analysis}

This section involves the analysis of the data obtained from the experiments conducted. The purpose of these experiments was to determine the performance of the CPE algorithm developed. The performance of the algorithm was measured in terms of its ability to accurately predict canine pose under USAR field conditions. The CPE algorithm was assessed as a true or false test, where the result was determined either correct or incorrect, under specific conditions. These conditions included the CPE algorithm indicating the correct pose within a set time of the canine having completed the pose. The conditions are explained further in next paragraph.

\subsection{CPE algorithm success data analysis}

A pose was considered complete when the dog was no longer moving its legs, excluding the walking pose. If the canine moved its legs, it was considered to still be in the process of adjusting into the final pose position. The walking pose was defined as complete as long as the canine was not stopped. In either situation head movements or tail wagging were considered to be insignificant to the definition of a completed pose.

Clearly, the CPE algorithm must be robust enough to make correct predictions despite the added signal distortion due to spurious tail and head movements. The data collected was synchronized with the video of the canine performing the pose during the experiment and then was carefully analyzed. The time at which the pose was completed was determined by reviewing the frame by frame video footage. Once this was determined the CPE algorithm's estimated pose was compared to the actual canine pose within the time window. The timestamps in the file of the data collected from the CPE device during the experiments was used to match the pose estimation with the video footage. If the poses matched, the algorithm's prediction was classified as correct for that test run.

The time window was chosen based on the observed transition time a canine took to perform a pose determined through observation. Each pose had a different transition time, some are almost instantaneous, while others required a few seconds to complete. The walking pose had an instantaneous transition time, while the standing and sitting poses had transition times ranging from two to three seconds. The lying down pose had a higher transition time, averaging five seconds, as more parts of the dog's body were involved in the transition motion. As a result, the time window was defined as two seconds. If the algorithm's estimated pose was consistent with the canine pose in the video and the prediction was within the time window, the CPE algorithm was successful.

The number of times that the algorithm was correct or incorrect for each pose was tabulated and this was used to determine the accuracy of the CPE algorithm to predict canine pose. The algorithms success rate was determined for each of the poses, as well as for the overall success of the predictions. These results are discussed in the next section.

\subsection{CPE algorithm success results}

The success of the algorithm was determined by obtaining the same results under the same conditions. This provided insight into how versatile and robust the algorithm was and whether it could successfully predict poses for different dogs, including dogs that had not been previously experimented with. A total of five canines were tested, running through each of the poses five times. The accuracy of the CPE algorithm for each of the poses is shown in Table 3.1. The overall accuracy rate of the CPE algorithm was found to be $80 \%$. The algorithm successfully estimated the standing pose for all canines for each of the test runs. The standing pose was easily distinguishable from the other poses. The angles' amplitude change did not vary greatly, even between the different dogs. The walking pose did not depend heavily on the location of the sensors and the size of the canine compared to the sitting and lying poses. 
The longer the canine's body, the greater the distance between the accelerometers attached to the dog, which increased the angle readings for the sitting pose. There was a greater variance among the canines' mean sitting pose angle. Regardless of the variations in the canines, we obtained a prediction success rate of $92 \%$ for the sitting pose.

Table 3.1. CPE algorithm accuracy

\begin{tabular}{|l|c|c|}
\hline \multicolumn{1}{|c|}{ Canine Pose } & Correct & Incorrect \\
\hline Standing & 25 & 0 \\
\hline Sitting & 23 & 2 \\
\hline Standing \& Sitting & 21 & 4 \\
\hline Walking & 9 & 16 \\
\hline Lying Down & 17 & 8 \\
\hline
\end{tabular}

The algorithm was not as successful in determining the walking pose as compared to the other poses. The algorithm predicted the walking pose only $36 \%$ of the time. The walking pose angles varied from canine to canine. Some of the USAR dogs had a calm walk, with a minimal bouncing up and down, where as other dogs walked around with a lot more bounce and energy. The algorithm was not robust enough to accurately estimate this pose across all the USAR canines.

Examining the lying down pose results, the algorithm had a $68 \%$ success rate. This pose was at times estimated to be sitting when the canine was in mid-pose. Synchronizing the data with the video enabled us to determine what contributed to this frequent false prediction. The dog's first sat down on their hind legs this is shown in Figure 3.1. They then proceeded to slide their front legs forward until their body lay parallel to the ground, shown in Figure 3.2.

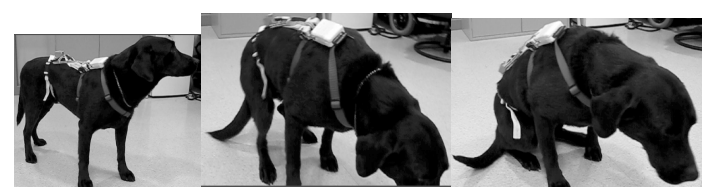

Figure 3.1. USAR canine DARE lying down video sequence part 1

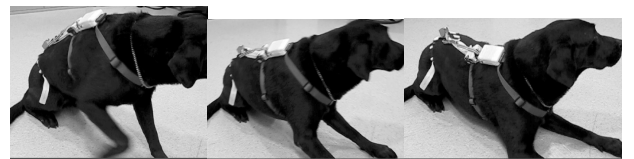

Figure 3.2. DARE lying down video sequence part 2
In some cases the canines would twist their hind legs so that the back end of their body was lying down sideways, see Figure 3.3 and Figure 3.4. The algorithm had a difficult time distinguishing between these orientations, which were more complex to determine and calculate.

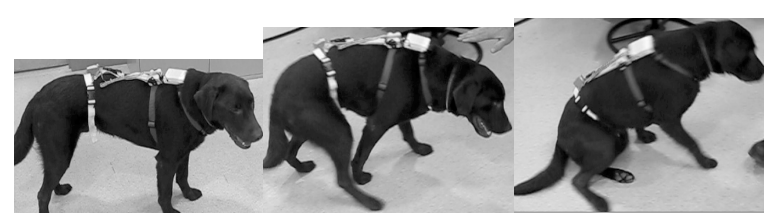

Figure 3.3. USAR canine Raker lying down video sequence part 1

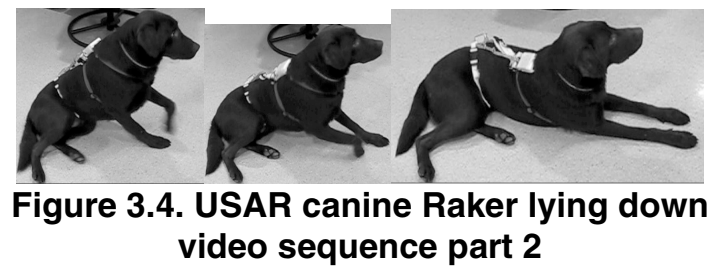

Another common incorrect estimation was for the lying down pose, which was estimated to be the standing pose. When standing, the accelerometers were horizontal as they were when the canine was lying down. This is one of the challenges that may be overcome with the addition of different sensors to the CPE device, this is discussed in section 6 , under future work.

\section{Conclusion}

This research contributes a potential solution for providing additional situational awareness for USAR operations. Emergency first responders, search managers and canine handlers all stand to benefit from the use of the CPE system. It could contribute to decreasing search times and increasing the number of lives saved in urban disasters.

The CPE algorithm was developed to determine canine pose from the angles measured on the search canines in real-time while conducting searches. The rule-based or heuristic algorithm computed angles and other metrics to predict the canine's pose from the incoming sensor data. Experiments were conducted to test the ability to determine angles from acceleration readings. The angle readings collected were similar for each of the poses even when tested on different USAR dogs.

The CPE algorithm achieved a high success rate for certain poses; however, other poses were not successfully determined. An overall success rate of 
$76 \%$ was obtained by the algorithm. The success rates for the individual standing, sitting, walking, and lying poses was $100 \%, 92 \%, 36 \%$, and $68 \%$ respectively. When testing a continuous sequence of standing and sitting poses, the algorithm was accurate $84 \%$ of the time. The walking and lying poses were difficult to determine as individual canines generally performed these poses differently.

The algorithm was robust enough to successfully estimate pose for all canines experimented on. It also predicted pose successfully when tested on new canines that were not part of the primary series of calibration experiments. The algorithm was capable of handling any added signal noise from the stray head and tail wagging movements of the canines.

\section{Future work}

\subsection{Additional sensors}

A force sensor or pressure pad could be used to improve the accuracy of the lying down pose. This would allow for the detection of the canine's abdomen lying on the ground. An alternative sensor that could be used for this purpose would be an ultrasonic sensor to determine the distance between the canine and the ground. When used outdoors this sensor is not very accurate.

The CPE algorithm uses the sensor data to determine the gravity vector and calculate the direction of the canine's movement. With respect to the walking pose this presents a unique challenge, as this assumption is only valid when the canine is not in motion. This is the reason for the CPE algorithm's high failure rate for the walking pose. When the canine walks there are additional impact forces being read by the sensors in addition to the directional acceleration and deceleration of the dogs body in motion.

To increase the CPE algorithm's success rate in estimating the walking pose, additional sensors could be added. Inertial Navigation Units, deal with similar situations of reading motion. These units use a combination of accelerometers and gyroscopes, in order to handle these cases. By adding a gyroscope to the CPE device the CPE algorithm could possibly increase the walking pose estimation success significantly.

\subsection{User interface design and functionality}

The user interface could be updated with an animation of a canine showing the poses. Also, increasing the animation image size could indicate its proximity and provide more insight and situational awareness to those that are simply interested in knowing the pose rather than interpreting data to deduce the pose.

\section{References}

[1] J. Tran, A. Ferworn, C.Ribeiro, M. Denko, "Enhancing Canine Disaster Search", IEEE SoSE 2008, June 2-5, 2008, Monterey, CA, USA.

[2] A. Ferworn, G. Hough, R. Manca, B. Antonishek, J. Scholtz, and A. Jacoff, Expedients for Marsupial Operations of USAR Robots, in IEEE International Workshop on Safety, Security and Rescue Robotics (SSRR06). Gaithersburg, MD, USA, 2006.

[3] M. Wolfe, A study of police canine search teams as compared to officer search teams, Canine Training Articles, T.U.S.P.C. Association (Editor), 1993, http://www.uspcak9.com/training/policesearchteams.shtml

[4] J. Casper and R. R. Murphy, Human-robot interactions during the robot-assisted urban search and rescue response at the world trade center, IEEE Trans Systems, Man and Cybernetics, Part B. Vol. 33, no. 3, pp. 367-385, 2003.

[5] Watanabe, S. and Izawa, M. and Kato, A. and RopertCoudert, Y. and Naito, Y., A new technique for monitoring the detailed behaviour of terrestrial animals: a case study with the domestic cat. Applied Animal Behaviour Science, Vol. 94, no. 1/2, pp. 117-131, 2005,

[6] http://www.petsmobility.com/petscell,accessed 04/01/08.

[7] Rogers, M.D ., Police Force! An Examination of The Use of Force, Firearms and Less-Lethal Weapons by British Police. The Police Journal, Vol 76, no. 3, pp. 189-203, 2003.

[8] Scholtz, J. and Antonishek, B. and Young, J., Evaluation of a human-robot interface: development of a situational awareness methodology. System Sciences, 2004. Proceedings of the 37th Annual Hawaii International Conference, pp. 130138,2004

[9] Yanco, H.A. and Drury, J., Where Am I? Acquiring Situation Awareness Using a Remote Robot Platform. IEEE Conference on Systems, Man and Cybernetics, 2004

[10] C.Ribeiro, A. Ferworn, M. Denko, J. Tran, C. Mawson, "Wireless Estimation of Canine Pose for Search and Rescue", IEEE SoSE 2008, June 2-5, 2008, Monterey, CA, USA.

[11] C. Ribeiro. "Canine Pose Estimation Using Wireless Networks", Thesis, University of Guelph, December 2008, Canada. 